\title{
Measurement of density fluctuations using digital holographic interferometry in a standing wave thermoacoustic oscillator.
}

\author{
Guillaume PENELET ${ }^{a}$, Mathieu LECLERCQ ${ }^{a}$, Thibault WASSEREAU ${ }^{a}$, \\ Pascal PICART ${ }^{\mathrm{a}}$ \\ ${ }^{a}$ Laboratoire d'Acoustique de l'Université du Maine, UMR CNRS 6613, Avenue Olivier \\ Messiaen, 72085 Le Mans Cedex 9, FRANCE
}

\begin{abstract}
This paper describes an optical set-up for measuring density fluctuations associated to acoustic oscillations in a thermoacoustic prime-mover. A timeresolved, full-field holographic interferometry technique is used, which enables to measure the optical phase difference between a reference beam and a probe beam passing through the acoustic resonator. The paper first presents the experimental set-up and the processing of holograms from which the instantaneous variations of the gas density along the line of sight of the probe beam are obtained. Then, the measurement technique is applied to the analysis of density fluctuations in the neighborhood of the heated side of a stack in a standing wave thermoacoustic prime mover during the transient regime of wave amplitude growth. The experimental results reveal that there exists very significant entrance effects, which lead to the generation of higher harmonics as well as mean (time-averaged) mass rarefaction in the vicinity of the stack termination. Finally, a short discussion is provided, based on a simplified modeling of higher harmonics generation in temperature associated to the oscillations of an inviscid gas through the stack, but the model fails in explaining the magnitude of the phenomena observed.
\end{abstract}

Keywords: Thermoacoustics, Digital Holography

Email address: guillaume.penelet@univ-lemans.fr (Guillaume PENELET) 


\section{Introduction}

A first approach of acoustical phenomena in fluids usually involves considering the wave process in terms of pressure and velocity fluctuations around an equilibrium state, and there exists different means to measure those quantities, such as microphones, Laser Doppler Velocimetry or Particle Image Velocimetry. However, density (as well as temperature) fluctuations always accompany pressure fluctuations, and it is sometimes worth considering to measure those density fluctuations, notably when analyzing unsteady heat and mass transfer phenomena involved in thermoacoustic engines.

The operation of thermoacoustic engines is governed by the thermoviscous interaction between gas oscillations and the solid frame of a porous material, referred to as a stack $[1,2]$. This stack is usually connected to heat exchangers, and inserted within an acoustical resonator. When the thermoacoustic engine is operated as a prime-mover, the application of a temperature gradient along the stack axis leads to the generation of self-sustained acoustic oscillations at the frequency of the most unstable mode ; when the thermoacoustic engine is operated as a heat pump, the sustain of resonant gas oscillations by an adequate source leads to advective heat transport by sound along the stack axis. The design and the development of thermoacoustic engines already has a three-decade history[3] and there exists several examples of devices able to reach high performances $[4,5,6,7,8,9]$. However, despite their simplicity in terms of geometry, thermoacoustic engines are not very well understood due to the complexity and the variety of the phenomena saturating the acoustic wave amplitude. Among these nonlinear phenomena are the ones impacting unsteady heat and mass transfer through the thermoacoustic core, such as acoustic streaming [10], or hydrodynamic/thermal entrance effects occuring notably at the stack termination (vortex shedding[11], transitional turbulence[12], nonlinear temperature fluctuations[13]).

Because of the complexity of the phenomena involved in thermoacoustics, it is useful to develop adequate measurement techniques allowing to get more information than the one a microphone could provide. Laser Doppler Velocimetry and Particle Image Velocimetry provide information about the gas parcel velocity and have been used previously to characterize acoustic streaming $[14,15,16,17]$ or vortex shedding processes $[18,19,20]$, while cold wire anemometry[21] has been used to characterize the nonlinear temperature fluctuations in the vicinity of the stack termination. In this paper, attention is focused on the development of another measurement method 
allowing to get information about density fluctuations from the variations of the optical index of the gas. This technique basically consists of an interferometer in which optical phase variations are obtained from a full-field, time-resolved digital holography technique.

Thanks to the development of high resolution CCD cameras and the increasing power of computers, digital holographic/interferometric measurements are nowadays under development and have already been used to analyze various processes of vibration kinematics [22, 23] or fluid mechanics[24], but only a few works have been made to characterize the density fluctuations associated to an acoustic process. There exists pioneering works performed fifteen years ago by Wetzel and Herman[25, 26, 27], in which analog holography has been used to measure temperature fluctuations at the end of a couple of parallel plates submitted to a high amplitude acoustic field. Interferometric techniques have also been used recently to characterize different classes of thermoacoustic phenomena, like sound generation by unsteady heat release within a flame[28], or the so-called piston effect generated in a small cell filled with critical $\mathrm{CO}_{2}[29]$, but these techniques only allowed the analysis of local (i.e. non full-field) density fluctuations. It is therefore worth considering to pursue the development of these techniques to get further information about various thermoacoustic phenomena, and more generally about some physical processes in which the derivation of density from pressure is not straightforward.

The device under consideration in this paper [see Fig. 1(a)] is a quarterwavelength thermoacoustic prime-mover, which simply consists of a straight duct, closed at one end, and equipped with a stack submitted to a temperature gradient. In the following, the measurement of density fluctuations by digital holography are performed in the vicinity of the hot side of the stack, during the transient regime of wave amplitude growth. The experimental setup and the data processing are presented in section 2. Experimental results are presented in section 3 , which enable to confirm that complicated heat and mass transfer processes are involved near the stack end. A discussion is provided in section 4, based on the comparison between experimental results and a simplified model developed by Gusev et al. [13]. 


\section{Experimental apparatus and data processing}

\subsection{Experimental apparatus}

A sketch of the thermoacoustic device under consideration in this study is presented in Fig. 1(a). It is composed of a glass tube $(49 \mathrm{~cm}$ in length, inner diameter $D_{i}=52 \mathrm{~mm}$, outer diameter $D_{o}=60 \mathrm{~mm}$ ) open at one end and closed by a rigid plug at the other end. Inside the tube is inserted the cylindrical stack (48 $\mathrm{mm}$ in length), whose diameter fits the inner diameter of the tube. This stack is made up of a 600 CPSI (cells per square inch) ceramic catalyst with numerous square channels of section $0.9 \times 0.9 \mathrm{~mm}$. The side of the stack facing the plug is heated using an electrical resistance wire (Nichrome wire, $36 \mathrm{~cm}$ in length, $0.25 \mathrm{~mm}$ in diameter, resistivity $7 \Omega / \mathrm{ft}$ ) regularly coiled through the stack end, and connected to a DC electrical power supply. Sound is captured using a $1 / 4$-in. condenser microphone (model GRAS, type 40BP) flush-mounted through the plugged end of the resonator. Note that a photograph of this device can also be found in ref. [30] ; it is worth mentioning that the presence of heater does not affect the optical measurements presented in the following, because the Nichrome wire is mounted flush inside the stack so that the laser beam used for digital holography does not pass through the heater.

In this study, the position of the stack along the resonator's axis is fixed at a distance $d=24 \mathrm{~cm}$ from the rigid plug. For this configuration, the onset of self-sustained thermoacoustic oscillations occurs as soon as the heat power supplied by the Nichrome wire exceeds the critical value $Q_{\text {onset }} \approx 20 \mathrm{~W}$ (note that this value of $Q_{\text {onset }}$ depends on the stack position [31]). The frequency $f \approx 171 \mathrm{~Hz}$ of acoustic oscillations corresponds to the quarter wavelength resonance, which means that $f \approx c_{0} / 4 L$, where $c_{0} \approx 344 \mathrm{~m} / \mathrm{s}$ stands for the adiabatic sound speed at room temperature. Previous studies of the same device[30, 31, 32] have clearly shown that, despite of its very simple geometry, this thermoacoustic oscillator can exhibit complicated dynamics of wave amplitude growth/saturation which are not reliably reproduced by theoretical/numerical modeling. It is therefore the objective of this study to perform holographic interferometry to analyze the refractive index variations in a window localized near the stack, where the temperature gradients are the highest, and to gain further insight on the physical processes controlling the saturation of the thermoacoustic instability.

A sketch of the optical set-up used to perform the measurement of density fluctuations close to the heated side of the stack is presented in Fig. 1(b). 
This set-up basically consists of a Fresnel interferometer enabling to measure the optical phase difference between a reference beam and a measurement beam passing through the acoustic resonator. This phase difference is caused by the variations of the refractive index within the resonator, due to both heat transport (notably the slow variations of the temperature field caused by heating) and acoustic (onset of self-sustained oscillations) processes. The light source is a laser source (Cobolt Flamenco, optical wavelength $\lambda=660$ $\mathrm{nm})$. The laser beam is split into a reference beam and a probe beam, and both beams are then expanded and bundled to parallel rays by a collimating lens. The probe beam passes through the acoustic resonator next to heated side of the stack, as indicated in Fig. 1(a). Then, the interference between the reference and the probe beam are captured by two CCD sensor (thanks to a 50/50 cube), namely a CCD camera and a high speed CMOS sensor [see Fig. 1(b)]. The CCD camera (model Hamamatsu ORCA-3CCD C7780-10) is used for analyzing slow temperature variations preceding the onset of selfsustained oscillations: the CCD sensor samples the interferogram at a rate of $1 \mathrm{im}$./s with $1344 \times 1024$ pixels sized $6.45 \mu \mathrm{m}$. The high speed CMOS sensor (Phantom V5.1) is used for analyzing the rapid fluctuations of the density occuring after the onset of self-sustained oscillations. This sensor is triggered when the peak amplitude of acoustic pressure exceeds the threshold value of $100 \mathrm{~Pa}$, thanks to the signal provided by the microphone. Interferograms are then sampled during 4 seconds at $1000 \mathrm{im}$./s with $1024 \times 1024$ pixels sized $14.8 \mu \mathrm{m}$. Note that the above mentioned choice is intrinsically related to the performance of the high speed camera, which sets the bounds of both time and space resolution, so that a compromise has to be found to get the best sampling rate with a maximum number of pixels. Here, the sampling frequency of $1 \mathrm{kHz}$ has been chosen so as to satisfy the Shannon-Nyquist criterium up to the second harmonic of thermoacoustic oscillations.

\subsection{Data processing}

The quantity of interest given by the optical set-up is the optical phase between the probe beam and the reference beam, which is related to the refractive index variation near the stack. From the optical set-up and adjustment of the beam splitter cube, digital holograms including spatial carrier frequencies (off-axis holography) are recorded and processed. The introduction of the spatial carrier frequency by the cube leads to the recording of one hologram at each instant, since there is no need for phase shifting [33]. This provided a single-shot and real-time capability to the experimental set-up to 
investigate acoustic phenomena. Fig. 2 shows the digitally recorded hologram with the left part of the image being close to the heat border of the stack. For any optical wavelength, the recorded image plane hologram can be expressed as [34, 35]:

$$
H(x, y)=O_{0}(x, y)+R(x, y) O^{*}(x, y)+R^{*}(x, y) O(x, y)
$$

where $O(x, y)$ and $R(x, y)$ are the object and the reference wave respectively. For convenience $R(x, y)$ can be represented with unitary amplitude. Accounting for the spatial carrier modulation introduced by the set-up, the hologram at optical wavelength $\lambda$ can be written as

$$
H(x, y)=O_{0}(x, y)+O(x, y) e^{2 i \pi\left(u_{r} x+v_{r} y\right)}+O^{*}(x, y) e^{-2 i \pi\left(u_{r} x+v_{r} y\right)},
$$

where $2 i \pi\left(u_{r} x+v_{r} y\right)$ is the spatial carrier phase modulation along the $x-y$ coordinates of the set of reference axis [see Fig. 1(b,c)], $O_{0}=|O(x, y)|^{2}+$ $|R(x, y)|^{2}$ is the zero order diffraction and we consider $O=a_{\lambda} e^{i \varphi_{\lambda}}$. The Fourier transform of Eq. (2) leads to [36]:

$$
\tilde{H}(u, v)=\tilde{O}_{0}(u, v)+\tilde{C}\left(u-u_{r}, v-v_{r}\right)+\tilde{C}^{*}\left(-u-u_{r},-v-v_{r}\right)
$$

where $\tilde{C}$ and $\tilde{O}_{0}$ are respectively the Fourier transform of $O$ and $O_{0}$, and where $(u, v)$ stand for the spatial frequencies.

Figure 3 shows the spatial frequency of the digital hologram. The zero order diffraction corresponds to central spot of the spectrum and the two complex conjugated orders of (3) are localized symmetrically. The order of interest is filtered using a binary filter centered at spatial frequencies $\left(u_{r}, v_{r}\right)$ indicated by the red cross. The dashed line indicates the frequency limits of the filter. If the spatial frequencies $u_{r}$ and $v_{r}$ are correctly adjusted, the three orders are well separated in the spatial frequency spectrum. By applying a bandwidth limited filter $(\Delta u \times \Delta v$ wide) around the spatial frequency $\left(u_{r}, v_{r}\right)$, and after filtering and inverse 2D Fourier transform, one gets the object complex amplitude[37]:

$$
C(x, y) \cong\left[a_{\lambda}(x, y) e^{i \varphi_{\lambda}(x, y)} e^{2 i \pi\left(u_{r} x+v_{r} y\right)}\right] * h(x, y)
$$

where $*$ means convolution product and where

$$
h(x, y)=\Delta u \Delta v e^{2 i \pi\left(u_{\lambda}+v_{\lambda}\right)} \operatorname{sinc}(\pi \Delta u x) \operatorname{sinc}(\pi \Delta v y)
$$


is the impulse response corresponding to the filtering applied in the Fourier domain. The spatial resolution is related to $1 / \Delta u$ and $1 / \Delta v$ respectively in the $\mathrm{x}-\mathrm{y}$ axis. In addition the phase recovered with Eq. (4) includes the spatial carrier modulation that has to be removed. This is achieved by multiplying $C$ by $e^{-2 i \pi\left(u_{r} x+v_{r} y\right)}$. Note that if the image of the test section is not in focus, the refocusing can be performed using the angular spectrum transfer function to get an in-focus image $[37,38]$. After the reconstruction process, both the optical phase and the amplitude can be estimated from Eq. (4):

$$
\begin{array}{r}
\varphi_{\lambda}(x, y)=\arctan \left(\frac{\Im[C(x, y)]}{\Re[C(x, y)]},\right. \\
a_{\lambda}(x, y)=\sqrt{(\Re[C(x, y)])^{2}+(\Im[C(x, y)])^{2}}
\end{array}
$$

where $\Re$ and $\Im$ respectively stand for the real and the imaginary parts of a complex number. In Fig. 4, a sample phase map is extracted and shown : the phase is calculated modulo $2 \pi$ and exhibits phase jumps. The curvature of the isophase lines observed in Fig. 4 can be attributed, at least partially, to the refraction of optical beam through the cylindrical glass tube. The effect of the glass tube can however be suppressed by substracting the phase map of Fig. 4 with the one obtained at a reference state, e.g. before switching on the heat supply.

When the test section is modified, for example due to heating and/or acoustic oscillations, a refractive index variation is induced along the object beam within the duct, and this modifies the optical path and then the optical phase. The phase change $\Delta \varphi_{\lambda}=\varphi_{\lambda}-\varphi_{\lambda}^{\text {ref }}$ between a current state $\varphi_{\lambda}$ and the reference state $\varphi_{\lambda}^{r e f}$, namely the one without heating and acoustic oscillations, is related to the associated difference of optical index as follows

$$
\Delta \varphi=\frac{2 \pi L(z)}{\lambda}\left(\langle n\rangle-\left\langle n_{r e f}\right\rangle\right)
$$

where the notation $\langle\ldots\rangle$ refers to an average along the beam path through the waveguide [length L(y), see Fig. 1(c)]. The mean refractive index $\langle n\rangle$ itself is related to the mean density along the line of sight, according to the Gladstone-Dale relation:

$$
\langle\rho\rangle=\frac{2}{3 \hat{r}}(\langle n\rangle-1),
$$


where $\hat{r}=0.1506 \times 10^{-3} \mathrm{~m}^{3} \mathrm{~kg}^{-1}$ is the specific refractivity of air at $\lambda=$ $607 \mathrm{~nm}$ (this parameter is almost independent of the optical wavelength, here $\lambda=660 \mathrm{~nm})$. As a result, the fluid density $\langle\rho\rangle(x, y, t)$ can calculated from the phase difference $\Delta \varphi_{\lambda}$ using Eqs. (8) and (9). Calculating the phase difference between each recorded instant leads to modulo $2 \pi$ phase map related both to temperature and density variations. Figure 5 shows such a phase map which cannot be used before phase unwrapping. Phase unwrapping consists in removing the $2 \pi$ phase jumps, so as to recover a continuous phase variation around the field of view [39]. Figure 6 shows the unwrapped phase map obtained after processing of phase from fig. 5, which itself allows to draw a map of the average density $\langle\rho\rangle$.

\section{Experimental results}

The experimental results presented hereafter are obtained for a heat power supply fixed to $Q=25.1 \mathrm{~W}\left(Q>Q_{\text {onset }}\right)$. The first interferogram obtained at time $\mathrm{t}=0 \mathrm{~s}$ is defined as the reference state from which the phase difference $\Delta \varphi_{\lambda}(x, y)$ is calculated. The DC power supply is then switched on, which leads around time $\mathrm{t}=75 \mathrm{~s}$ to the onset of self-sustained acoustic oscillations at frequency $f \approx 171 \mathrm{~Hz}$. Both the regime of heating before onset and that of wave amplitude growth are analyzed using digital holography (using the CCD camera and the high speed CMOS sensor, respectively).

First of all, we analyze the evolution of density during the heating stage. Before the onset of the thermoacoustic instability, the pressure is constant and equals the atmospheric pressure at room temperature, $P_{0}=1.015 \times$ $10^{5} \mathrm{~Pa}$. Due to this, it is straightforward to obtain the temperature field $\left\langle T_{0}\right\rangle(x, y)$ averaged along $\mathrm{z}$ through the probe beam [see Fig 1(c)], using the ideal gas law

$$
P_{0}=\langle\rho\rangle R\langle T\rangle,
$$

where $R=287.058 \mathrm{~J} . \mathrm{kg}^{-1} \cdot K^{-1}$ stands for the specific gas constant of air. In Fig.7, the measured increase of $\langle T\rangle$ as a function of time is presented for different positions $x$ along the resonator's axis. More precisely, the data presented are calculated from the interferograms obtained with the CCD sensor, and correspond to the evolution with time of the temperature $\langle T\rangle$, which itself is averaged (for each sample interferogram) through a small zone of cross-sectional area $10 \times 50$ pixels along $\mathrm{x}$ and $\mathrm{y}$, respectively, the latter zone being itself centered at some position $x$ along the resonator's axis. The 
data presented are obtained for 5 different positions, from $x=0.3 \mathrm{~mm}$ up to $x=6 \mathrm{~mm}$ [the reference position $x=0$ corresponds to the heated side of the stack, see Fig. 1(a)]. From the results presented in Fig.7, one can clearly see the gradual increase of the temperature, as well as the establishment of a steep, negative temperature gradient. It is worth reminding that the magnitudes of the temperature presented in Fig. 7 are z-averaged magnitudes along the line of sight [see Fig. 1(c)], and that these temperatures are lower than the one we can measure using a thermocouple along the resonator's axis; this simply means that the temperature field is not uniform through the cross sectional area of the resonator. At time $t \approx 75 \mathrm{~s}$, the onset of self-sustained thermoacoustic oscillations clearly appears on the data depicted in Fig. 7. However, as soon as the onset occurs these data are not reliable because the sampling rate $(1 \mathrm{~Hz})$ is much lower than the acoustic frequency, and also because equation (10) can no longer be used to calculate temperature variations from density variations (the pressure being no longer constant). As a result, only the temperature data on the left of the vertical dashed line in Fig. 7 are worth considering, and finally the data obtained once the onset of self-sustained oscillations has occurred will be the ones captured with the high speed camera, while only density fluctuations (instead of temperature fluctuations) will be presented in the following.

In Fig. 8, the time-resolved variations of both acoustic pressure (a,a') and density (b-e) are presented. Acoustic pressure fluctuations are measured by the microphone at position $x=d$ during the whole transient regime, while density fluctuations are measured during 4 seconds once the high speed camera is triggered at time $t \approx 77 \mathrm{~s}$. The time variations of density are presented for four axial positions, namely $x_{1}=1.3 \mathrm{~mm}, x_{2}=3 \mathrm{~mm}, x_{3}=4.7$ $\mathrm{mm}$, and $x_{4}=6.4 \mathrm{~mm}$. As clearly seen in Fig. 8, the starting point and the duration of data acquisition coincides with the final stage of wave amplitude growth, up to its saturation to a finite amplitude (after an overshoot process which occurs around $t \approx 78.3 \mathrm{~s}$ ). The same dynamical range (i.e. from 0.7 up to $\left.1.2 \mathrm{~kg} \cdot \mathrm{m}^{-3}\right)$ is used in the presentation of the densities $\langle\rho\rangle\left(x_{i}, t\right)$ in Figs. 8(b-e). This enables to see clearly that the amplitude of density oscillations strongly depend on the observation point $x_{i}$ : these amplitudes are much higher at positions $x_{1}$ and $x_{2}$ than at positions $x_{3}$ and $x_{4}$. It is worth pointing out that the distance $x_{4}-x_{1}$ (about $5 \mathrm{~mm}$ ) is much lower than the acoustical wavelength (about $2 \mathrm{~m}$ ), which means that the observed variations are not related to standard acoustic propagation. In other words, if the measured density fluctuations were only related to the presence of a standing 
acoustic wave, then the amplitude at position $x_{4}$ should be the same as the one at position $x_{1}$. Therefore, the fluctuations of density presented in Fig 8 (c-e) emphasize the presence of entrance effects. We use this terminology (entrance) to refer to some effects associated to the abrupt transition at the ends of the stack. These entrance effects have two natures: one is related to the geometrical singularity at the end of the stack which leads to flow separation (and possibly vortex shedding) and viscous dissipation, while the other one is related to a singularity in terms of heat transfer due to the fact that a gas parcel oscillating next to the end of the stack experiences an abrupt transition between a polytropic motion (inside the stack) and an essentially adiabatic motion (outside the stack).

Further analysis of the experimental data is provided in Fig. 9, where the slow variations (as compared with the period of oscillations) of the mean density are plotted as a function of time during the four seconds of measurements. More precisely, the sliding average (denoted $\rho_{m}$ ) over 17 acoustic periods (with a $50 \%$ time-recovery) computed from the raw data $\langle\rho(x, t)\rangle$ is presented as a function of time and for different positions $x$. The results of Fig. 9 clearly exhibit a significant variation of the mean density, which also strongly depends on the distance from the stack. There is indeed a significant decrease of $\rho_{m}$ occuring around $t \approx 78.5 \mathrm{~s}$ (i.e. around the overshoot of acoustic pressure amplitude): this decrease can reach about $7 \%$ of the initial value (at time $t=77 \mathrm{~s}$ ) at a distance of about 1.8 or $2.4 \mathrm{~mm}$ from the stack, while it is less than $2 \%$ at $x=0.2 \mathrm{~mm}$ and at $x=5.8 \mathrm{~mm}$. Such experimental results therefore indicate that entrance effects are responsible for a mean (i.e. non-oscillating) nonlinear mass transport phenomenon localized in the vicinity (but not in direct contact) of the stack termination.

It is also interesting to analyze the spectral components of density fluctuations, notably because previous theoretical [13] and experimental [21] studies have shown that the stack termination should be responsible for higher harmonics generation in temperature fluctuations (even if pressure fluctuations should be monochromatic). It is therefore expected that the latter impact on temperature fluctuations should also modify the spectral content of density fluctuations. In Fig. 10 we present the amplitudes of the fundamental and the second harmonic components of density fluctuations (obtained from the Fast Fourier Transform of raw data during the four seconds of data acquisition), as a function of the distance $x$ from the hot side of the stack. Additionally, a rough estimate of the quasi-static (i.e. slowly varying) component of density fluctuations is also presented in Fig 10: this quasi-static amplitude 
is evaluated from the absolute difference between the initial mean density $\rho_{m}(x, t=77 \mathrm{~s})$ and its time-average $\overline{\rho_{m}}$ during the four seconds of data acquisition. It is also interesting to evaluate the expected peak amplitude that density fluctuations should have if both the stack and the temperature gradient were absent. Such a reference amplitude, denoted $\rho_{a d}$. in the following, can be obtained from the microphone signal and from the assumption that an adiabatic process of plane wave propagation occurs along the resonator. This leads to the following formula

$$
\rho_{a d}=\overline{P_{\text {mic. }}} \cos (k d) / c_{0}^{2}
$$

where $\overline{P_{m i c}}$ stands for the peak amplitude of the microphone signal averaged along the four seconds of data acquisition, and where $k=2 \pi f / c_{0}$ is the acoustical wave number. This reference amplitude of the fundamental component is (almost) independent of position $x$ since the width of optical beam is here much lower than the acoustical wavelength. From the results presented in Fig 10, there exist a significant distorsion of density fluctuations in the vicinity of the stack, since the amplitude of the second harmonic is not small compared to that of the fundamental (especially around $x \approx 4-5$ $\mathrm{mm}$ where the amplitude of the second harmonic can reach about $20 \%$ of the fundamental). It is also clear that the spatial distribution of both the fundamental and the second harmonic components strongly depend on the distance $x$ from the stack. From the comparison of the measured amplitudes and the one predicted from the microphone signal (dashed line), we can conclude once again that the results exhibit the impact of entrance effects, since far from the stack, the measured amplitude of the fundamental component tends towards the predicted amplitude $\rho_{a d}$ while the amplitudes of both the second harmonic and the quasi-static components tend towards zero.

\section{Discussion}

The results of Fig. 10 confirm previous experimental[11, 18, 21], numerical[11, 21] or analytical[13] studies, whose main conclusions state that there exists complicated heat and mass transport phenomena next to the ends of a stack. However, a new point here is that we measured density fluctuations (instead of velocity or temperature fluctuations), but also that the device we studied is a self-sustained thermoacoustic oscillator (instead of a stack at room temperature submitted to an acoustic wave by an external sound source), which 
notably means that there exists also a steep temperature gradient at the location where measurement are processed. Moreover, we did not proceed to measurement during a controlled steady-state regime of acoustic oscillations, but instead during the transient regime of wave amplitude growth. As a result, it is not straightforward to compare our experimental data with any analytical/numerical model describing a situation identical to the one we analyzed.

An attempt is made in the following to compare our experimental data with the simplified analytical model provided by Gusev et al. [13]. This model enables to evaluate the distorsion of temperature fluctuations experienced by gas parcels oscillating through the stack termination. Because such a problem is generally not tractable analytically, some simplifications are required and it is therefore assumed (1) that the fluid is inviscid, (2) that the stack plates are infinitely thin and have an infinite thermal conductivity, (3) that the static temperature is constant at room temperature, (4) that the axial conduction in the fluid is neglected while (5) transverse heat conduction inside the stack is taken into account via a phenomenological parameter (equivalent to using a Newton's law of cooling with an arbitrarily fixed heat transfer coefficient). Finally, assuming that the fluid is submitted to a standing acoustic wave at angular frequency $\omega=2 \pi f$ in the frame of the linear acoustics approximation (and therefore without any distorsion of the pressure and velocity field next to the stack termination in the inviscid fluid), and accordingly with the notation used in ref.[13], this results in the following equation for a dimensionless gas temperature $\theta$ [see ref. [13] for more details]:

$$
\frac{\partial \theta}{\partial \tau}+\sin \tau \frac{\partial \theta}{\partial \xi}=\sin \tau-\frac{\theta}{R},
$$

where $\tau$ and $\xi$ are dimensionless variables defined as $\tau=\omega t$ and $\xi=x / u$, and where $u$ stands for the peak amplitude of the gas displacement at the location of the stack. In Eq. (12), the dimensionless temperature is defined as $\theta=T^{\prime} / T_{c}$ where $T_{c}$ is a characteristic amplitude of adiabatic temperature fluctuations in a standing wave, while the parameter $R$ is a dimensionless relaxation time used to account for the thermal coupling between the gas and the stack walls. In the following, we will consider that inside the stack $(\xi \leq 0)$ one has $R=1$ while outside the stack $(\xi \geq 0)$ it tends towards infinity: this assumption amounts to considering that the distance between two stack plates is such that the thermoacoustic process is optimum $(R=1)$ 
while the process is purely adiabatic outside the stack $(R \rightarrow \infty)$. Note that both the characteristic amplitudes of gas displacement $u$ and temperature fluctuations $T_{c}$ can be obtained from the (time-averaged) peak amplitude of pressure fluctuations measured by the microphone as follows (accordingly with ref. [13]):

$$
\begin{aligned}
u & =\frac{k \overline{P_{m i c .}}}{\rho_{0} \omega^{2}} \tan (k d), \\
T_{c} & =-\frac{\overline{P_{\text {mic. }}}}{\rho_{0} C_{p}} \cos (k d) .
\end{aligned}
$$

As shown by Gusev et al. [13], Eq. (12) can be solved analytically. In the following, we provide the solution in the domain $\xi \geq 0$, which is written as:

$$
\theta=-\frac{1}{2}\left[\xi+\cos \tau+\sin \left(\tau_{+}\right)\left(1-\frac{e^{-\tau_{+}}}{\sinh \left(\tau_{+}\right)}\right)\right]
$$

if $|\xi+\cos \tau| \leq 1$, with $\tau_{+}=\arccos (\xi+\cos \tau)$, and

$$
\theta=-\cos \tau
$$

if $|\xi+\cos \tau| \geq 1$. This solution is valid outside the stack region $\xi \geq 0$ up to $\xi=2$ (i.e. up to the distance by which an oscillating gas parcel may cross the stack during a cycle) and for $\tau \in[-\pi, \pi]$. From the Fourier series expansion of the solution $\theta(\xi, \tau)$, it is then straightforward to get the amplitudes of the mean (time-averaged), the fundamental and the harmonic components of $\theta$ as a function of its mean position $\xi$. Finally, turning back to dimensional amplitudes $T^{\prime}(x, t)$, it is possible to plot the predicted spectral components of temperature fluctuations as a function of the distance $x$ from the hot side of the stack. To that purpose we use $d=24 \mathrm{~cm}, \overline{P_{\text {mic. }}} \approx 548 \mathrm{~Pa}$, and $f=171.2 \mathrm{~Hz}$ as input parameters, which corresponds to the experimental data, and which results in $\left|T_{c}\right| \approx 1.17 \mathrm{~K}$ and $u=1.2 \mathrm{~mm}$. The results obtained for the fundamental, the harmonic and the time-averaged ( $\sim$ quasistatic) components of temperature fluctuations are presented in Fig 11(a). Such results reveal that, because of the singularity in term of heat transfer at the stack termination, there exists a significant generation of the second harmonic as well as a mean component of temperature, while the fundamental component itself varies significantly along the axis. It is predicted from this model that these entrance effects extend up to twice the displacement 
amplitude $u$, and that only the fundamental component remains for $x \geq 2 u$ (with a peak amplitude $T_{c}$ ). The spatial distribution of both the fundamental and the second harmonic of temperature fluctuations presents some similarities with the experimental data of Fig. 10: one can notably remark that in both cases there exists a minimum for the amplitude of the second harmonic which is located at some position which roughly corresponds to the maximum of the fundamental. However, the data presented in Fig. 11(a) cannot be directly compared to those of Fig. 10, since the former deals with temperature while the latter deals with density. Therefore, the theoretical variations of the spectral components of density fluctuations as a function of position $x$ are presented in Fig. 11(b). These variations are calculated from the temperature fluctuations using the linearized ideal gas law:

$$
\rho^{\prime}(x, t) / \rho_{0}=p^{\prime}(x, t) / P_{0}-T^{\prime}(x, t) / T_{0}
$$

in which the pressure fluctuations are perfectly harmonic (as initially assumed in the model) and almost independant of axial position $x$ (since $2 u \ll c_{0} / f$ ):

$$
p^{\prime}(x, t) \approx \overline{P_{\text {mic. }}} \cos (k d) \cos (\omega t) .
$$

For a better readability we did not use the same dynamical range in Fig. 11(b) as the one used in Fig. 10, but it is worth pointing out that the range of variation of $\rho^{\prime}$ is much higher in experiments (i.e. up to $\approx 0.05 \mathrm{~kg} . \mathrm{m}^{-3}$ ) than in the model (up to $\approx 0.005 \mathrm{~kg} \cdot \mathrm{m}^{-3}$ ). There is therefore a significant discrepancy between the simplified model and the experimental results, and the model underestimates the maximum amplitudes of the fundamental (factor $\times 10$ ), the second harmonic (factor $\times 5$ ) and the quasi-static (factor $\times 8$ ) components. Also, while the model predicts that entrance effects extend up to $\approx 2.5 \mathrm{~mm}$, the experiments show that the entrance effects extend up to $\approx 6 \mathrm{~mm}$. Such an extension of the impact of entrance effects beyond the distance $2 u$ has already been reported by Berson et al.[21] both numerically and experimentally (from the measusurement of $T^{\prime}$ using cold wire anemometry), and can be explained (at least partially) by accounting for the axial conduction within the fluid in Eq. (12). However, the large discrepancy in terms of amplitudes as well as the differences in terms of spatial distribution (notably for the fundamental and the quasi-static components) is not explained. The model used here is based on numerous assumptions, but it has the merits to emphasize that the observed experimental results cannot be only explained by an abrupt transition in terms of heat transfer at the 
stack interface. We believe that the more drastic assumption made here is that of an inviscid fluid, which means that aerodynamical effects like flow separation, vortex shedding, or possibly jet-driven streaming are not considered, and the resulting nonlinear heat and mass transport by the above mentioned effects is not considered. Both aerodynamical entrance effects and the presence of a steep temperature gradient should therefore be considered in the modeling to get a better agreement. Such a task falls out of the scope of this paper, and the important point is that the experiments reveal that the impact of entrance effects on density is very significant. As a result, the possible impact of such entrance effects on the dynamics of wave amplitude growth in thermoacoustic devices is worth considering for future works.

\section{Conclusion}

This paper presents an interferometric holographic measurement technique which has been successfully applied to the measurement of density fluctuations associated to acoustic oscillations in a thermoacoustic primemover. To our knowledge, such a technique has not been applied to acoustics or thermoacoustics, except for the analogous holography technique used by Wetzel and Herman in the late 90's [25, 26, 27]. This measurement technique appears to be a simple and promising way to get further insight into the operation of thermoacoustic engines (and, maybe, into other acoustical problems) as a complementary tool to other techniques like Particle Image Velocimetry or Cold Wire Anemometry. Although the results presented in this paper remain unexplained because of the lack of an appropriate model, they reveal that the stack termination generates both gas rarefaction and higher harmonics generation in the vicinity of the stack termination. The magnitude of the effect is such that, beyond the only dissipation of acoustical work it causes (minor losses), the question of its impact on the thermoacoustic process (wave amplification and thermoacoustic heat flux within the stack) should be considered cautiously for future works.

\section{Acknowledgments}

This research was funded from the French National Agency for Research under grant agreement ANR 2010 BLAN 030201. 


\section{Bibliography}

[1] G.W. Swift, Thermoacoustics: A unifying perspective for some engines and refrigerators, Acoustical Society of America, Melville NY (2001).

[2] N. Rott, Thermoacoustics, Adv. Appl. Mech. 20 (1980) 135-175.

[3] J. Wheatley, T.J. Hofler, G. W. Swift, A. Migliori, Understanding some simple phenomena in thermoacoustics with applications to acoustical heat engines, Am. J. phys., 53 (1985) 147-162.

[4] G.W. Swift, Analysis and performance of a large thermoacoustic engine, J. Acoust. Soc. Am. 92 (1992) 1551-1563.

[5] S.L. Garrett, J.A. Adeff, T.J. Hofler, Thermoacoustic refrigerator for space applications, J. of Thermophysics and Heat Transfer, 7 (1993) 595-599.

[6] S. Backhaus, G.W. Swift, A thermoacoustic Stirling heat engine, Nature 399 (1999) 335-338.

[7] S. Backhaus, E. Tward, M. Petach, Traveling-wave thermoacoustic electric generator, Appl. Phys. Lett. 85 (2004) 1085-1087.

[8] M.E.H. Tijani, S. Spoelstra, A high performance thermoacoustic engine, J. Appl. Phys. 110 (2011) 093519.

[9] Z. Wu, G. Yu, L. Zhang, W. Dai, E. Luo, Development of a 3 kW doubleacting thermoacoustic Stirling electric generator, Applied Energy 136 (2014) 866-872.

[10] S. Boluriaan, P.J. Morris, Acoustic streaming: from Rayleigh to today, Int. J. of Aeroacoustics 2 (2003) 255-292.

[11] Ph. Blanc-Benon, E. Besnoin, O. Knio, Experimental and computational visualization of the flow field in a thermoacoustic stack, C. R. Mec. 331 (2003) 1724.

[12] C. Scalo, S.K. Lele, L. Hesselink, Linear and nonlinear modelling of a theoretical travelling-wave thermoacoustic heat engine, J. Fluid Mech., 766 (2015) 368-404. 
[13] V. Gusev, P. Lotton, H. Bailliet, S. Job, M. Bruneau, Thermal wave harmonics generation in the hydrodynamical heat transport in thermoacoustics, J. Acoust. Soc. Am. 109 (2001) 84-90.

[14] M.W. Thompson, A.A. Atchley, Simultaneous measurement of acoustic and streaming velocities in a standing wave using laser Doppler anemometry, J. Acoust. Soc. Am. 117 (2005) 1828-1838.

[15] S. Moreau, H. Bailliet, J.C. Valire, Measurements of inner and outer streaming vortices in a standing waveguide using laser Doppler velocimetry, J. Acoust. Soc. Am. 123 (2008) 640-647.

[16] C. Desjouy, G. Penelet, P. Lotton, J. Blondeau, Measurement of acoustic streaming in a closed-loop traveling wave resonator using Laser Doppler Velocimetry, J. Acoust. Soc. Am. 126 (2009) 2176-2183.

[17] I. Reyt, H. Bailliet, J.C. Valire, Experimental investigation of acoustic streaming in a cylindrical wave guide up to high streaming Reynolds numbers, J. Acoust. Soc. Am. 135 (2014) 27-37.

[18] A. Berson, Ph. Blanc-Benon, Nonperiodicity of the flow within the gap of a thermoacoustic couple at high amplitudes, J. Acoust. Soc. Am. 122 (2007) EL122-EL127.

[19] A.J. Jaworski, X. Mao, X. Mao, Z. Yu, Entrance effects in the channels of the parallel plate stack in oscillatory flow conditions, Exp. Therm. Fluid Sci. 33 (2009) 495-502.

[20] L. Shi, Z. Yu, A.J. Jaworski, Vortex shedding flow patterns and their transitions in oscillatory flows past parallel-plate thermoacoustic stacks, Exp. Therm. Fluid Sci. 34 (2010) 954-965.

[21] A. Berson, G. Poignand, Ph. Blanc-Benon, G. Comte-Bellot, Nonlinear temperature field near the stack ends of a standing-wave thermoacoustic refrigerator, Int. Journ. Heat Mass Transfer 54 (2011) 4730-4735.

[22] P. Picart, J. Leval, F. Piquet, J.P. Boileau, J.P. Dalmont, Analysis of Clarinet Reed Oscillations With Digital Fresnel Holography, Europ. J. Phys. Appl. Phys. 47 (2009) 12706. 
[23] M. Leclercq, M. Karray, V. Isnard, F. Gautier, P. Picart, Evaluation of surface acoustic waves on the human skin using quasi-time-averaged digital Fresnel holograms, Appl. Optics. 52 (2013) A136-A146.

[24] J.M. Desse, P. Picart, P. Tankam, Digital color holography applied to fluid and structural mechanics, Optics and Laser in engin., 50 (2012) $18-28$.

[25] M. Wetzel, C. Herman, Limitations of temperature measurements with holographic interferometry in the presence of pressure variations, Exp. Therm. Fluid Sci. 17 (1998) 294-308.

[26] M. Wetzel, C. Herman, Experimental study of thermoacoustic effects on a single plate Part I: Temperature fields, Heat Mass Transf. 36 (2000) $7-20$.

[27] M. Wetzel, C. Herman, Experimental study of thermoacoustic effects on a single plate Part II: Heat transfer, Heat Mass Transf. 35 (1999) 433-441.

[28] T. Leitgeb, T. Schuller, D. Durox, F. Giuliani, S. Koberl, J. Woitsetschlager, Interferometric determination of heat release rate in a pulsated flame, Combustion and flame 160 (2013) 589-600.

[29] Y. Miura, S. Yoshihara, M. Ohnishi, K. Honda, M. Matsumoto, J. Kawai, M. Ishikawa, H. Kobayashi, A. Onuki, High-speed observation of the piston effect near the gas-liquid critical point, Phys. Rev. E 74 (2006) 010101.

[30] G. Penelet, T. Biwa, synchronization of a thermoacoustic oscillator by an external sound source, American Journ. Phys., 81 (2013) 290-297.

[31] G. Penelet, M. Guedra, V. Gusev, T. Devaux, Simplified account of Rayleigh streaming for the description of nonlinear processes leading to steady state sound in thermoacoustic engines, Intern. Journ. Heat Mass Transfer, 55 (2012) 6042-6053.

[32] M. Guedra, G. Penelet, P. Lotton, Experimental and theoretical study of the dynamics of self-sustained oscillations in a standing wave thermoacoustic engine, J. Appl. Phys. 115 (2014) 024504. 
[33] I. Yamaguchi, T. Zhang, Phase shifting digital holography, Optics Letters 22 (1997) 1268-1270.

[34] E. Cuche, F. Bevilacqua, C. Depeursinge, Digital holography for quantitative phase contrast imaging, Optics Letters 24 (1999) 291-293.

[35] U. Schnars and W. Jptner, Direct recording of holograms by a CCD target and numerical reconstruction, Appl. Opt. 33 (1994) 179-181.

[36] J. Desse, P. Picart, and P. Tankam, Digital three-color holographic interferometry for flow analysis, Opt. Express 16 (2008) 5471-5480.

[37] J. Li, P. Tankam, Z. Peng, and P. Picart, Digital holographic reconstruction of large objects using a convolution approach and adjustable magnification, Opt. Lett. 34 (2009) 572-574.

[38] P. Picart, P. Tankam, D. Mounier, Z. Peng, and J. Li, Spatial bandwidth extended reconstruction for digital color Fresnel holograms, Opt. Express 17 (2009) 9145-9156.

[39] D.C. Ghiglia, M.D. Pritt, Two-Dimensional Phase Unwrapping : Theory, Algorithms and Software, Wiley Ed., New York (1998). 

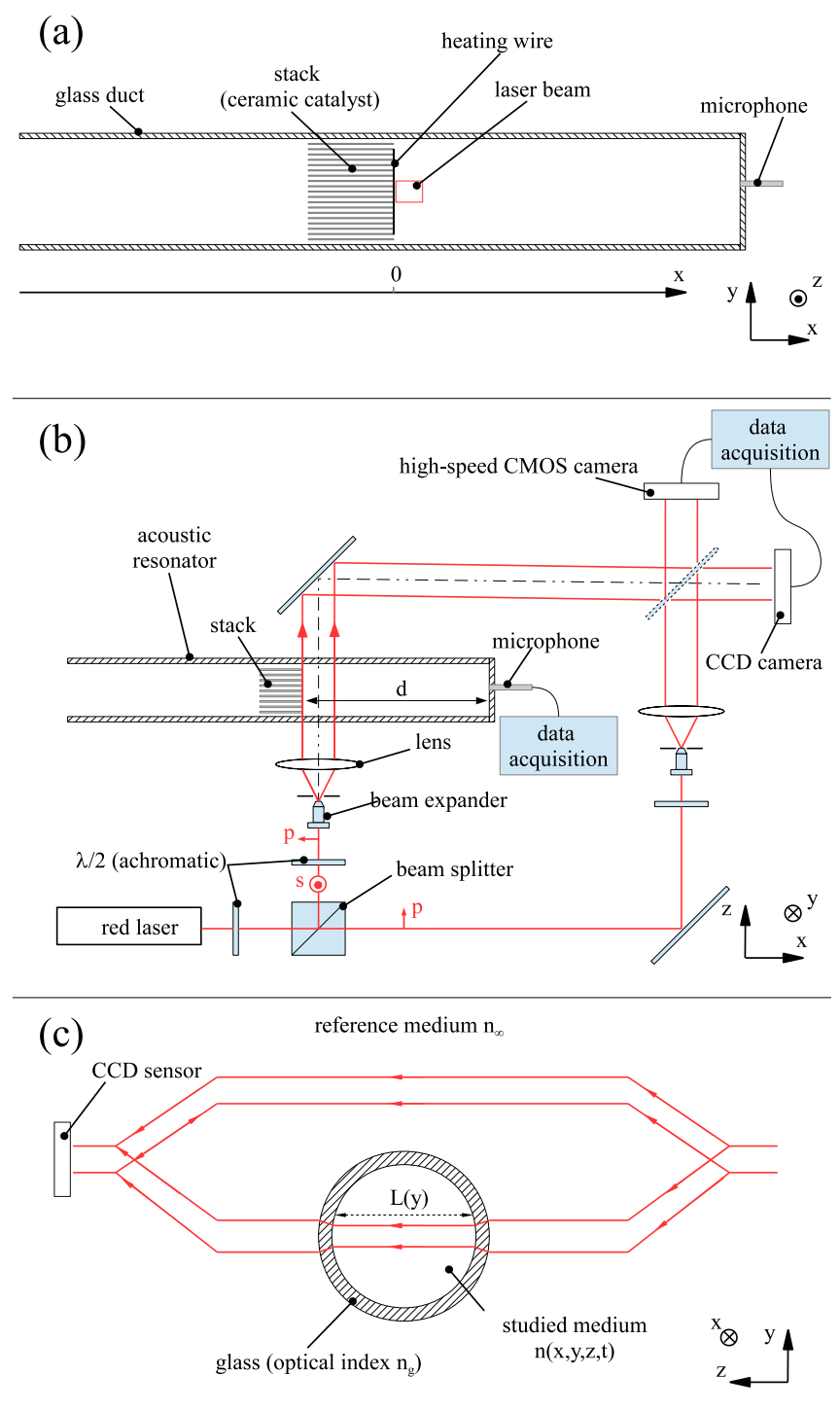

Figure 1: Sketch of the experimental apparatus, including the thermoacoustic oscillator (a) and the optical set-up (b) which basically consists of a reference beam and a measurement beam passing through the acoustic resonator (c) close to the heated end of the stack. 


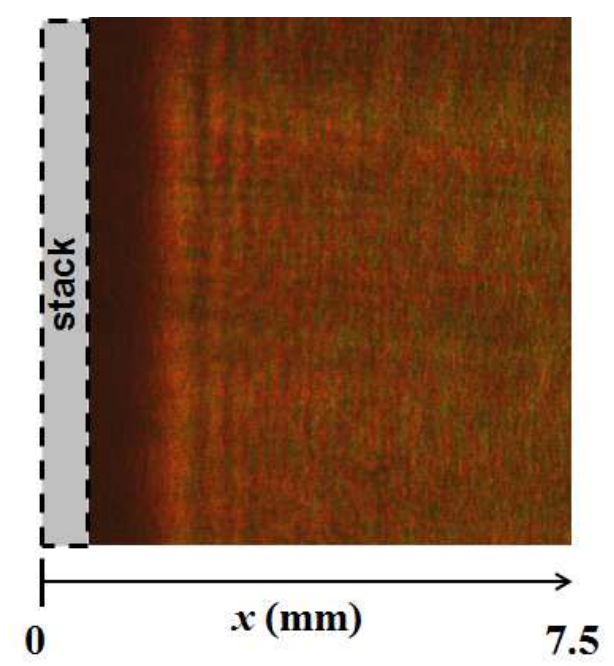

Figure 2: An example of a digitally recorded hologram. The stack is located on the left hand side of the picture.

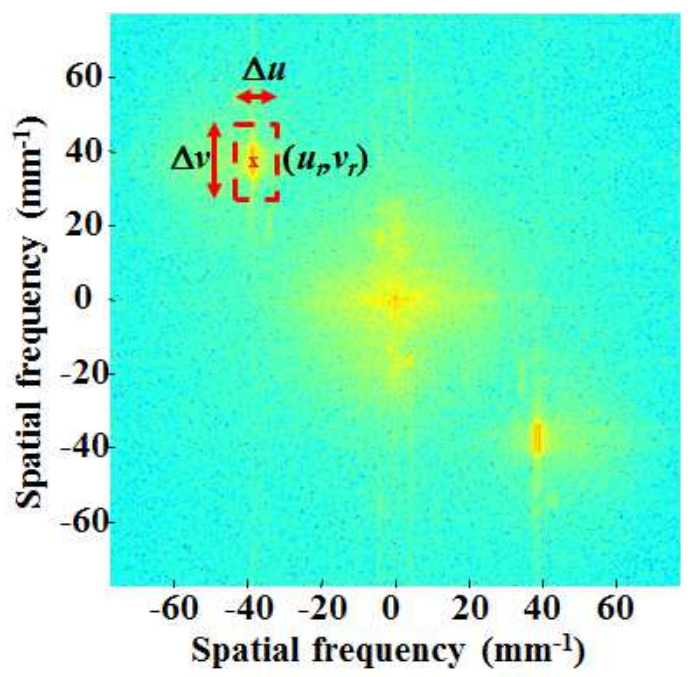

Figure 3: Spatial Fourier spectrum of the recorded hologram. 


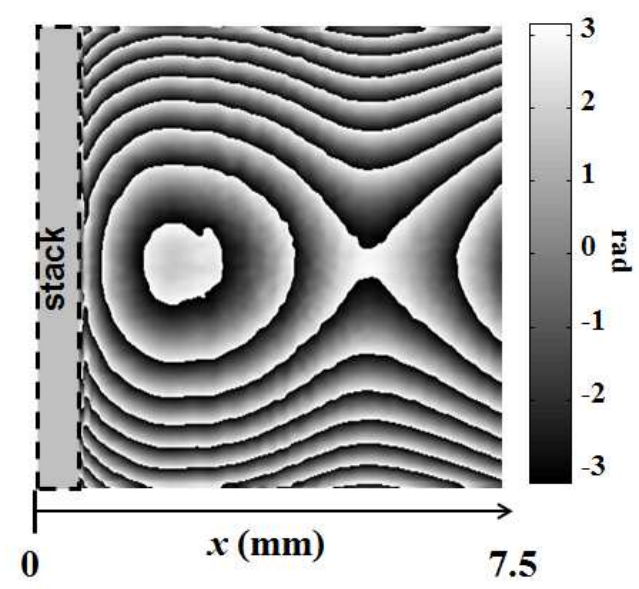

Figure 4: Phase map extracted from the filtered Fourier spectrum of Fig. 3

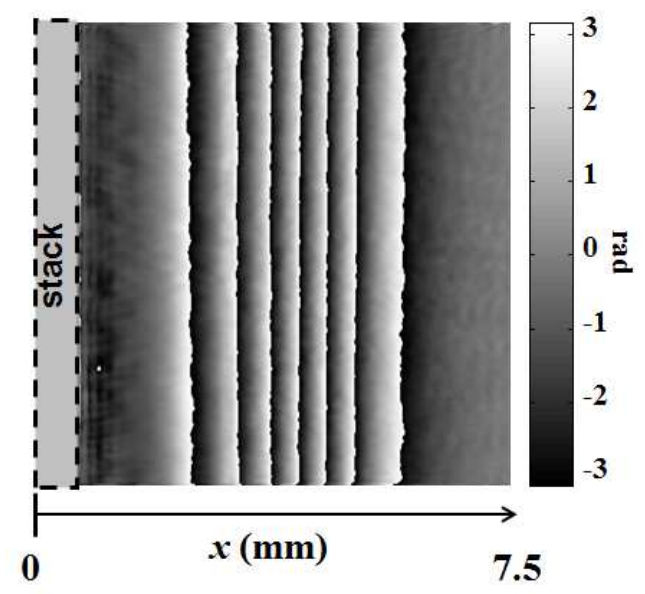

Figure 5: Phase difference $\Delta \varphi_{\lambda}$ between the current state and a reference state (e.g. neither heating nor acoustic wave) as a function of $x$ and $y$. 


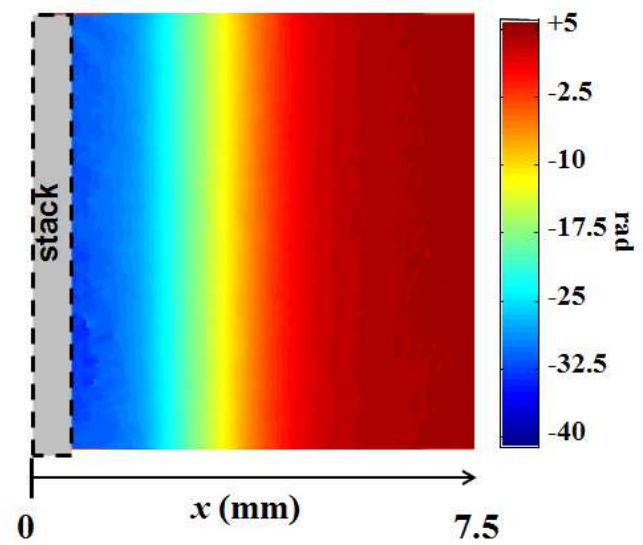

Figure 6: Phase difference $\Delta \varphi_{\lambda}$ between the current state and a reference state (e.g. neither heating nor acoustic wave) as a function of $x$ and $y$ after the process of phase unwrapping.

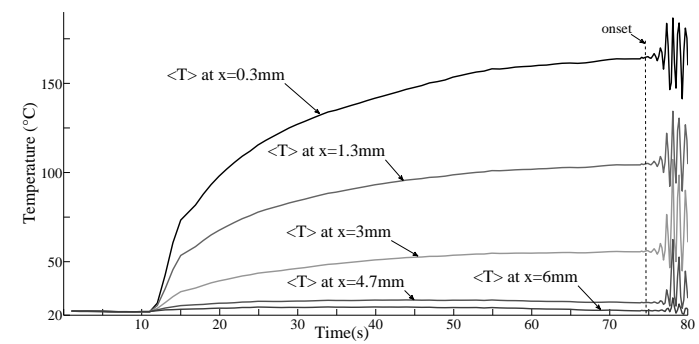

Figure 7: Variations as a function of time of the temperature next to the heated side of the stack during the process of heat supply, for different positions along the resonator's axis. These temperatures, obtained from digital holograms and the ideal gas law, are averaged temperature along the line of sight of the probe beam through the resonator. The reference position $x=0$ corresponds to the heated side of the stack. 

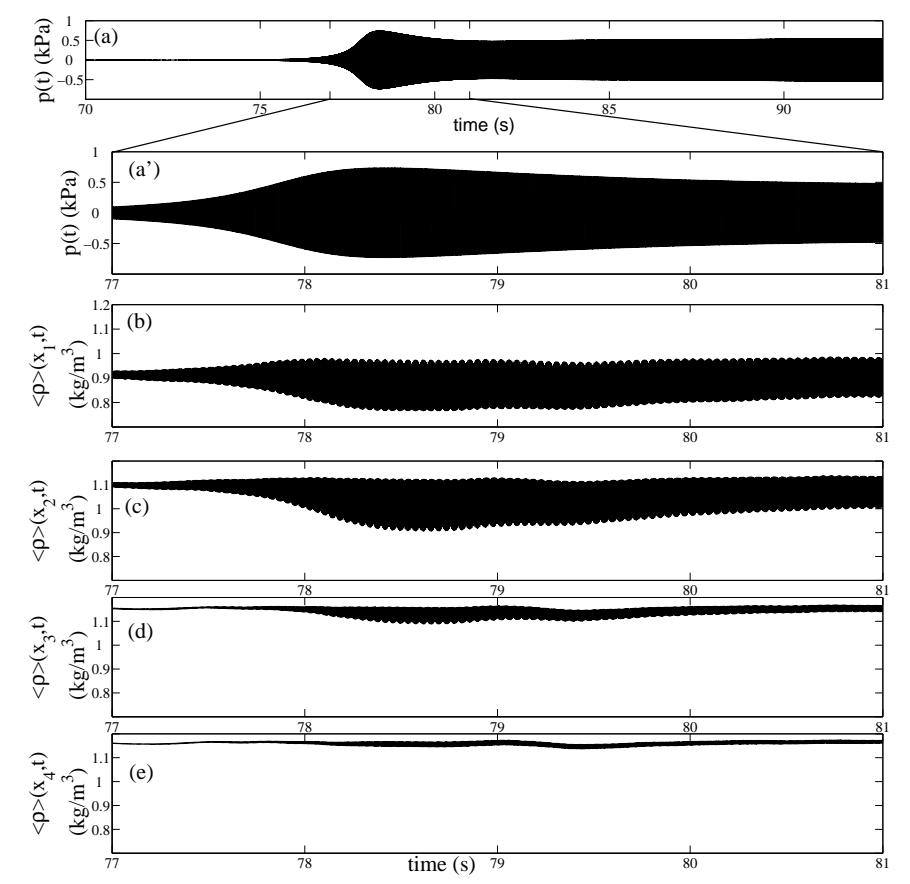

Figure 8: Acoustic pressure (a,a') and density (b-e) fluctuations as a function of time during the transient regime of wave amplitude growth. The measurement of density fluctuations $\rho\left(x_{i}, t\right)$ are processed during 4 seconds from the holograms sensed by the high speed camera at a sampling rate of $1 \mathrm{kHz}$. Axial positions $x_{i}$ correspond to $x_{1}=1.3 \mathrm{~mm}$, $x_{2}=3 \mathrm{~mm}, x_{3}=4.7 \mathrm{~mm}$, and $x_{4}=6.4 \mathrm{~mm}$.

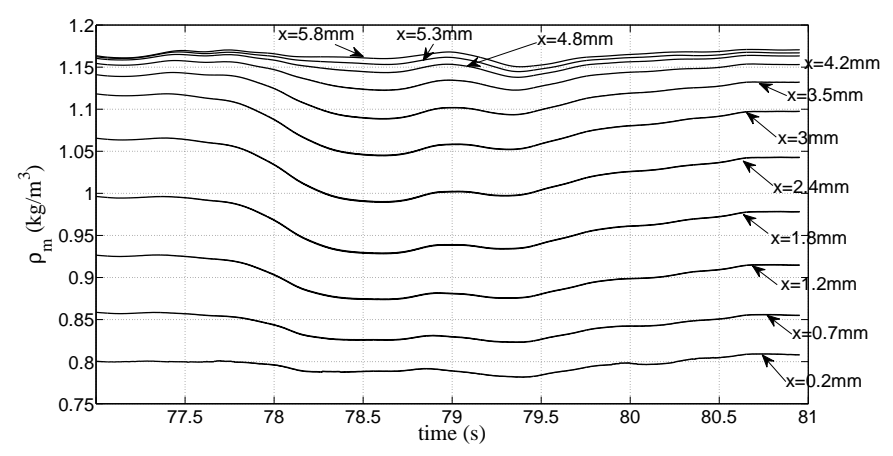

Figure 9: Quasi-static, acoustically induced variations of the mean density $\rho_{m}$ as a function of time and axial position $x(\mathrm{x}=0$ refers to the hot side of the stack). These quasi-static variations are evaluated from $\left\langle\rho^{\prime}\right\rangle(x, t)$ using a sliding average over 100 successive sample images (i.e. during $\approx 17$ acoustic periods) with a $50 \%$ recovery. 


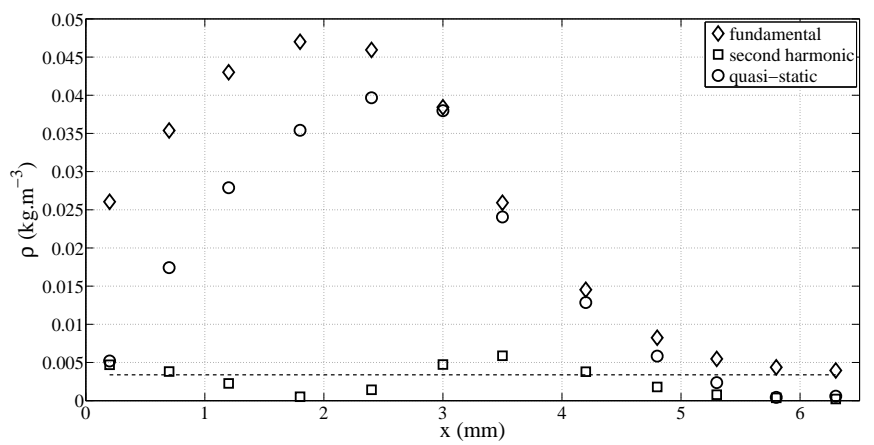

Figure 10: Amplitudes of the fundamental $(\diamond)$, the second harmonic $(\square)$ and the quasistatic (o) components of density fluctuations obtained in experiments as a function of axial position $x$. In addition, the horizontal dashed line corresponds to the peak amplitude of density fluctuations estimated from the microphone signal using the assumption of a linear and adiabatic process of acoustic propagation. Note that the evaluated peak amplitude of gas displacement for this configuration is $u \approx 1.2 \mathrm{~mm}$. 

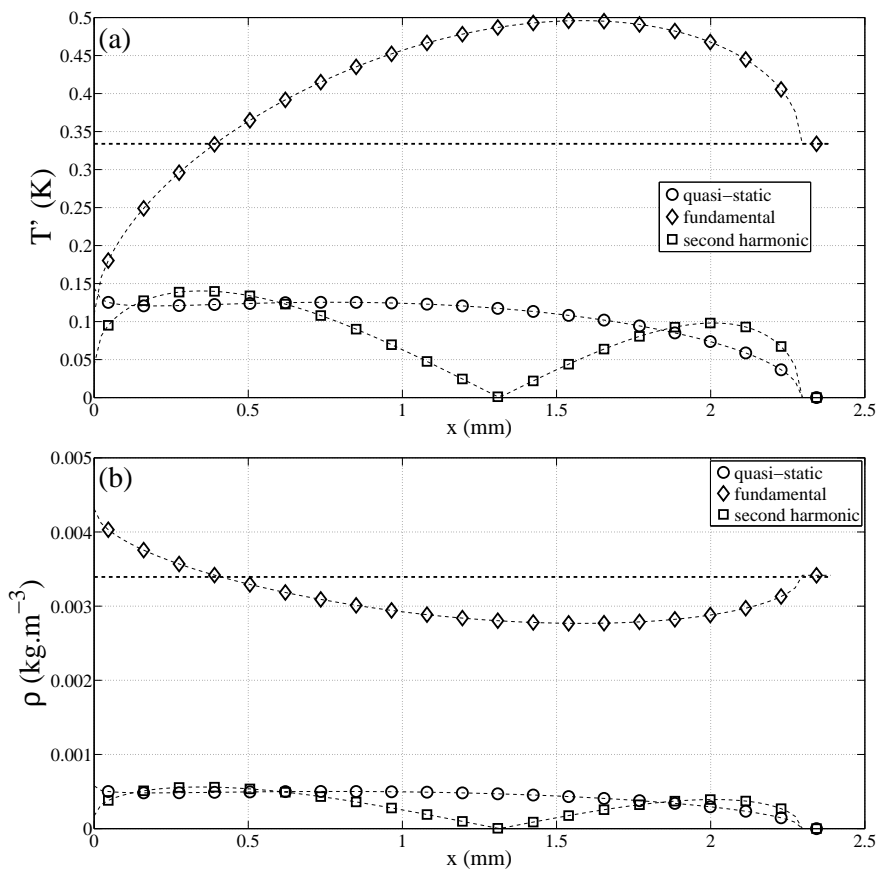

Figure 11: Theoretical amplitudes of the fundamental $(\diamond)$, the second harmonic $(\square)$ and the quasi-static (o) components of both temperature (a) and density (b) fluctuations as a function of axial position $x$. In addition, the horizontal dashed lines correspond to the peak amplitude of temperature (a) and density (b) fluctuations estimated from the microphone signal using the assumption of a linear and adiabatic process of acoustic propagation. Note that the evaluated peak amplitude of gas displacement for this configuration is $u \approx 1.2$ $\mathrm{mm}$. 